\title{
Reflets
}

Revue d'intervention sociale et communautaire

\section{L'aide : entre compassion et militantisme}

\section{Nérée St-Amand}

Volume 23, numéro 2, automne 2017

URI : https://id.erudit.org/iderudit/1043309ar

DOI : https://doi.org/10.7202/1043309ar

Aller au sommaire du numéro

Éditeur(s)

Reflets, Revue d'intervention sociale et communautaire

ISSN

1203-4576 (imprimé)

1712-8498 (numérique)

Découvrir la revue

Citer cet article

St-Amand, N. (2017). L'aide : entre compassion et militantisme. Reflets, 23(2),

225-232. https://doi.org/10.7202/1043309ar d'utilisation que vous pouvez consulter en ligne.

https://apropos.erudit.org/fr/usagers/politique-dutilisation/ 


\section{L'aide : entre compassion et militantisme}

\section{Nérée St-Amand, Ph.D.}

Professeur titulaire, École de service social, Université d'Ottawa

"Pour moi, le militantisme, ça fait partie de la vie. Ce n'est pas abstrait et ce n'est surtout pas juste un emploi. C'est vivre! »

François Saillant

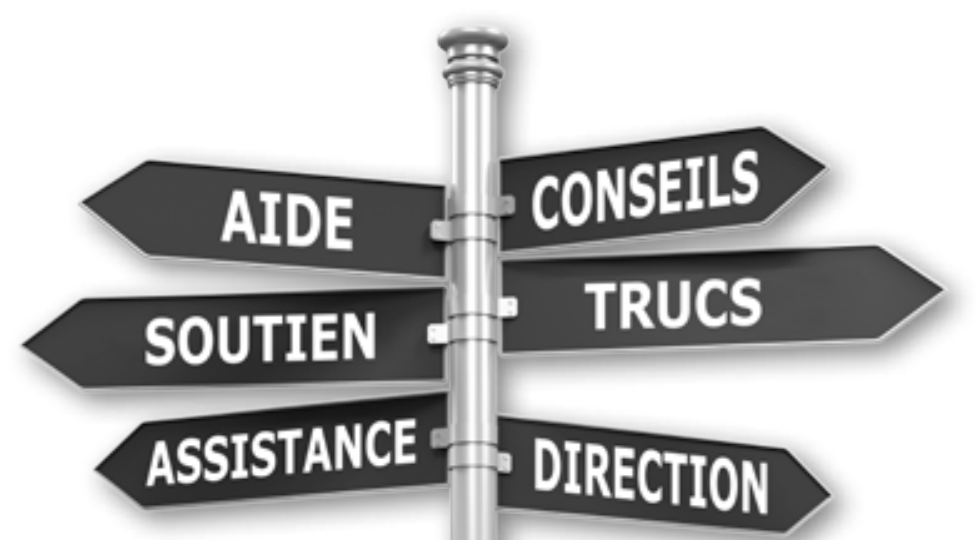

\section{Un brin d'histoire...}

Quand je me suis inscrit en travail social, à la fin des années 1970, c'était tout simplement pour aider les autres et, ce faisant, rejoindre cette génération de personnes rêvant à la fois de compassion, de charité et de justice. Les lieux et formes d'intervention étaient alors en train 
de changer radicalement, passant, en une décennie, des institutions religieuses aux réseaux professionnels qui s'implantaient à la suite du vide laissé par une Église chancelante. Au Nouveau-Brunswick, ma province natale, comme ailleurs, nous assistions à la mise en place de cette professionnalisation de l'aide orchestrée par l'État. En parallèle, mais souvent à l'écart, se développaient diverses initiatives communautaires basées sur le partage, la solidarité, le militantisme. Ces formes de pratique étaient souvent considérées comme marginales et non comme du "vrai » travail social. Mais dans l'ensemble, qu'importe les lieux et formes d'intervention, nous aidions les gens!

Aider! Un idéal tout simple, tout pur! Je faisais alors partie de cette génération de missionnaires désenchantés qui se cherchaient un champ de pratique autre que l'Église, institution qui se vidait alors et qui ne constituait plus une avenue acceptable pour les transformateurs sociaux. Car l'Église, bastion espéré du changement social, perdait ses troupes. Pour ma part, j'avais envisagé une carrière dans une communauté religieuse, mais j'avais constaté, comme plusieurs, la schizophrénie d'une Église aux discours et pratiques contradictoires. En cours de route, Ivan Illich m'a aidé à comprendre ces contradictions entre nobles discours et difficiles réalités.

À la suite de Vatican II en particulier, cette Église faisait face à de nombreux défis, comme la place des femmes ou encore le célibat des prêtres, l'avortement et l'homosexualité. Nonobstant les nombreuses contradictions dont j'étais aussi témoin et porteur, l'aide altruiste m'interpellait toujours, héritage de cette idéologie chrétienne. Je souhaitais consacrer ma vie à " aider " mon prochain. Délaissant le clergé, j’ai alors opté pour ce que je considérais comme la profession la plus proche de mes valeurs : le travail social.

Maintenant, après quarante ans de pratique et de recherche, je propose ici une réflexion qui se veut une remise en question de l'aide, ce concept central à notre profession. En partant de quelques observations, je tente de démontrer que le travail social est porteur de nobles possibilités mais aussi de profondes contradictions. Je souligne ainsi quelques interrogations que l'aide suscite pour ensuite présenter des pratiques basées sur des valeurs et principes qui s'éloignent de l'empathie charitable. Je souhaite ainsi participer à la construction d'une conception militante de l'aide qui s'éloigne à la fois de la psychologie et de nos héritages chrétiens pour proposer des pistes de réflexion axées sur la justice, l'écologie et la solidarité. 


\section{Aider, en toute naïveté}

Dans les années 1970, au sein d'une équipe de protection de l'enfance, aider une jeune mère célibataire enceinte était tout simple : il s'agissait de lui conseiller — sinon de lui imposer - de donner son enfant en adoption! De la sorte, nous apportions une aide évidente et généreuse à trois personnes au moins : la mère biologique qui ne pouvait pas garder son enfant, un couple adoptif qui voulait fonder une famille et un enfant "illégitime ». En plaçant l'enfant dans un contexte favorable à ce qu'elle ou il devienne une citoyenne ou un citoyen respectable, aux bonnes valeurs, nous faisions en sorte qu'elle ou il ne reproduise pas le parcours que sa mère biologique avait emprunté. Nos valeurs ressortaient éloquemment de cet exemple dans un contexte où l'aide semblait un geste aussi simple qu'évident! Cependant, il ne tenait pas compte des rapports de classe et de genre, des enjeux de pouvoir ni de la force des appareils idéologiques de l'État.

En appui à la pratique, la littérature sur l'aide était tout aussi florissante et convaincante que psychologisante. Les grands auteurs de la psychologie rogérienne, comportementale et plus tard systémique démontraient déjà les bienfaits indéniables de l'aide. La littérature en relation d'aide prenait une ampleur considérable, suscitait de grands intérêts et mobilisait les associations professionnelles, sans oublier les collèges, universités et centres de recherche. Et ce que l'État et ses appareils ne pouvaient gérer, les organismes caritatifs ou privés s'en chargeaient.

Aider, dans le contexte de ces premières observations, allait de soi : en aidant l'autre, nous faisions en sorte qu'elle ou il entre dans les rangs de l'idéologie dominante. Il n'était pas question de remettre en cause les principes sous-tendant cette idéologie; les " clients " restaient des " clients ", et les personnes aidantes, des agentes professionnelles de l'aide. Pour le novice que j'étais, tous ces acteurs et actrices constituaient une symbiose parfaite d'intérêts convergents et d'objectifs en parfaite harmonie. Qui plus est, l'entraide s'est rapidement ajoutée à nos champs d'intervention, et la magie de l'aide s'est vue multipliée du fait que, en aidant l'autre, on s'aidait soi-même. La littérature sur l'aide a constitué dès lors la vache à lait de la psychothérapie. Un discours rassurant, rempli de certitudes, comme le démontre cette citation :

Permettre à l'usager de reconquérir son autonomie, lui venir en aide et assurer sa protection sont des objectifs communs aux différentes catégories de travailleurs sociaux. Ceux-ci sont bien sûr confrontés dans leur pratique à des publics ayant des difficultés ou des handicaps spécifiques. Pour atteindre ces objectifs, il importe que l'accompagnement assuré par le travailleur social 
se fasse en respectant la dignité, les intérêts et le rythme de la personne prise en charge, ainsi que la confidentialité des informations la concernant. [Le soulignement est de moi.]

Melchior, 2011, p. 124

Nous assistons depuis à un foisonnement des formes et modalités de l'aide tant et si bien qu'en 2017, une simple recherche du mot " help » sur la toile fait ressortir plus de huit milliards de sites s'y consacrant. Facile d'en déduire que l'aide est à peu près omniprésente et considérée comme un incontournable. De plus, l'aide n'est pas facilement remise en question. En somme, l'aide s'est spécialisée, acquérant ainsi les lettres de noblesse d'une profession qui s'est graduellement détachée, du même coup, de certaines réalités comme la pauvreté et la violence en particulier. Cependant, quelques brèches laissaient poindre de profondes contradictions au niveau des analyses de genre, de classe et de race en particulier. Ces analyses permettaient de poser plusieurs questions comme les trois que je retiens ici : L'aide est-elle là pour soutenir les personnes défavorisées ou plutôt pour entretenir quelques mythes psychothérapeutiques et idéologiques? Pourquoi l'aide est-elle généralement individuelle, alors que la grande majorité des problèmes sont collectifs? Comment se fait-il que le travail social et les autres professions " thérapeutiques " s'intéressent si peu à la défense des droits des personnes et groupes opprimés?

\section{L'aide : passage du psychosocial au politique}

En posant ces questions, j’ai graduellement compris qu'il s'agissait d'un modèle d'aide basé sur la charité, la compassion, minimisant du même coup l'impact des inégalités socio-économiques et les conséquences structurelles de la pauvreté. En cours de route, j’ai émis l'hypothèse que le travail social privilégiait la même analyse que proposait l'Église en se basant sur l'expertise des personnes professionnelles et la non-reconnaissance du potentiel des pauvres de se prendre en main. En somme, une aide visant la conformité, une aide qui suggère que les gens ne peuvent se sortir de la misère sans une intervention professionnelle.

Dans un tel contexte, la plupart des programmes de formation et les intérêts des étudiantes et étudiants convergent dans le sens de privilégier une intervention individuelle pour répondre aux besoins sociaux. Les organismes d'aide, malgré un discours 
d'empowerment, encouragent souvent les gens à accepter leur situation bien plus qu'à la changer. Somme toute, au cours de mes années de pratique et de recherche, je suis devenu de plus en plus convaincu que les associations professionnelles, les groupes caritatifs, les communautés religieuses, les clubs sociaux n'avaient pas comme but véritable d'éliminer la pauvreté mais plutôt de la perpétuer. J'ai trouvé très difficile de poser de telles questions dans certains contextes de pratique, institutionnel entre autres, alors que, comme chercheur et comme professeur, ce genre de questionnement était plus acceptable. Par ailleurs, travailler avec des personnes victimes de systèmes oppressifs m'a aussi grandement aidé à comprendre autrement et à investir dans d'autres façons d'aider, dans d'autres formes d'aide. En fait, l'aide, en cours de route, a basculé du psychologique au politique.

\section{Quelques pratiques inspirantes}

Mais alors, comment aider? Certaines pratiques d'aide, émanant de contextes d'oppression et d'une analyse plus sociale, plus radicale, m'incitent à poser certaines questions visant à comprendre autrement. L'aide est-elle genrée? L'aide est-elle culturelle? L'aide se conjugue-t-elle de façon différente selon les classes sociales, les cultures, les lieux où on habite, l'histoire qu'on a apprise ou qu'on privilégie? Les quelques exemples qui suivent m'amènent à redéfinir la notion de l'aide, fondée sur une prise de conscience et une visée de changement social.

\section{Le refus global}

Ce manifeste, paru en 1948, ouvre la porte à une prise de conscience des immenses inégalités de pouvoir dans le Québec de Duplessis, certes, mais à maints autres lieux et niveaux également. Grâce à ce manifeste, le monde artistique et professionnel brise le silence de l'acceptation, refuse la soumission à l'autorité, ce qui ouvrira la voie à d'autres mouvements de contestation, en psychiatrie par exemple ou dans les luttes raciales et de genre.

\section{L'Abbé Pierre : « Vous, les riches »}

À partir du moment où il est entré chez les Capucins à 17 ans, le cheminement de l'Abbé Pierre change de façon radicale. Rapidement, ses interventions passent du caritatif au politique. Il n'hésite pas à contester l'ordre établi, même s'il faut pour ceci recourir à des pratiques radicales ou même illégales. Avec les années, son analyse devient virulente, et il nous interpelle lorsqu'il accuse les riches de "provocateurs de toutes violences ", 
dénonçant l'hypocrisie de leurs " mains d'inconscients ». Son discours résonnait avec mon parcours, et ses analyses mettant clairement les classes sociales au cœur de l'aide m’ont touché et éclairé.

\section{Les marches des femmes}

Au Canada, deux marches historiques des femmes, celle de 1995 (Du pain et des roses) et celle de 2000 (Marche mondiale des femmes contre la pauvreté et la violence faite aux femmes), constituent des exemples marquants de revendications des femmes. L'artisane du mouvement, Françoise David, propose alors un modèle d'aide qui, à l'exemple de Jane Addams, contribue à redéfinir l'intervention sociale axée sur sa dimension politique. Cette travailleuse sociale et militante engagée redéfinit ce qu'est l'intervention sociale basée sur la solidarité collective. Les inégalités de genre ressortent clairement, et j’ai mieux compris que la marche constitue une forme d'intervention trop peu explorée, mais combien efficace.

\section{Le refus de Rosa, Viola, Lia}

Rosa Parks est loin d'être la seule à dénoncer la ségrégation raciale, mais son nom évoque tout un mouvement de transformation des rapports basés sur la race et la classe en particulier. Sa lutte de 1955 symbolise cette posture que les personnes défavorisées peuvent prendre pour revendiquer : protester en restant assise, refuser de se soumettre, constitue une approche dont plusieurs se sont inspirés par la suite. Un événement similaire est survenu chez nous neuf ans plus tôt : Viola Desmond a connu un sort identique, en Nouvelle-Écosse cette fois. En 1946, alors qu'elle est en voyage d'affaires à New Glasgow, elle se présente au cinéma et achète un billet à l'étage principal, réservé aux Blancs. Quand les autorités lui demandent de bouger, elle refuse. Arrêtée, emprisonnée et condamnée, elle a continué ses luttes pour les populations noires, créant un réseau d'écoles de beauté y accueillant les personnes refusées par les autres écoles. Son refus d'accepter l'inacceptable a nécessité, soixante ans plus tard, une correction de l'histoire : l'effigie de Viola Desmond figure sur un timbre-poste canadien et sur le billet de dix dollars, non sans qu' elle ait reçu en cours de route un pardon inconditionnel et des excuses de la part des autorités provinciales.

Pour sa part, Lia Tran, une jeune Acadienne ayant reçu un diagnostic de schizophrénie, se révolte après des années de très grande souffrance, va voir son psychiatre et met fin au joug que constituait pour elle la psychiatrie. Elle refuse son diagnostic et ses traitements et sort du bureau en jurant de ne jamais y remettre les pieds. Ces femmes m'ont aidé à 
comprendre que le refus met en valeur la dignité des personnes et les changements de rapports de pouvoir lorsque cette stratégie est privilégiée.

\section{Les Autochtones d'Oka et leurs revendications territoriales}

Le scandale de la ville d'Oka a également constitué un moment fort de refus et de mobilisation. Le conflit a duré plusieurs mois et a divisé de nombreuses populations, autochtones et autres. Cet exemple de refus illustre le fait qu'une décision présentée comme toute simple par les autorités municipales a connu une escalade de tensions et d'attention médiatique au niveau international. Qui plus est, la défiance a marqué les négociations dans le contexte de cette revendication. Certains contextes de refus d'obtempérer aux autorités peuvent amener des situations de défiance justifiée.

L'aide, dans de tels exemples, commence par les personnes défavorisées elles-mêmes : un appel à la justice, à l'équité, sinon à la révolte contre des gens et des institutions qui sont censés aider. Ce faisant, ces actrices et acteurs démontrent que les systèmes ne sont pas immuables et que, sous l'effet de la contestation, ils deviennent plus flexibles. Pour ma part, ces personnes ont contribué à redéfinir l'aide empreinte de dignité. D'autres personnes, comme Jackie Vautour et Rosemary Brown par exemple, m'ont beaucoup marqué par leur détermination, leur sens de la justice et leur force de caractère. Enfin, les Autochtones, par leur sagesse millénaire, m’ont aidé à comprendre que la spiritualité n'est pas un volet ajouté à l'intervention sociale, mais qu'elle est au cœur de l'aide. Dans ce contexte, la roue de la médecine, les cercles de guérison ont nourri et inspiré mes pratiques et mes enseignements, car ils intègrent la spiritualité, les cercles de vie et le respect des générations, de l'écologie et des cultures.

En résumé, en me basant sur mes observations et mes pratiques d'intervention, je suggère que toute aide véritable risque d'être subversive, revendicatrice, basée sur une analyse politique, fondée sur une analyse des rapports de pouvoir et spirituelle. La revendication politique est à la base d'une aide véritable, et mes expériences m’ont convaincu que l'empowerment par et pour les personnes et les groupes victimes est la seule voie qui changera les rapports sociaux de l'aide. Les travailleuses et travailleurs sociaux peuvent appuyer ces personnes et ces mouvements communautaires, venir en renfort aux personnes défavorisées et affirmer leur capacité d'être des artisans de changements structurels et donc des agents d'aide véritable. Dans un tel contexte, le travail social est-il une profession d'aide? Ma réponse, après quarante ans de pratiques, de recherches et de questionnements, reste conditionnelle. Dans un premier temps, il nous faut prendre conscience de nos biais idéologiques, de nos valeurs ancrées et de nos lieux et 
formes d'engagement. Par la suite, si nous privilégions des modèles de militantisme et de conscientisation, si notre profession adopte des formes de pratique inspirées par Jane Addams, Rosemary Brown, les sages autochtones ou David Suzuki, si elle s'associe à des mouvements planétaires comme le Forum social mondial, si elle sait accepter que, comme l'Église et l'État, il a longtemps été un outil d'oppression, alors, tout devient possible, et l'aide peut devenir un outil de changement social, politique, idéologique, et accomplir ainsi sa mission véritable : devenir un outil de transformation sociale.

\section{Note}

1 Extrait d'une allocution prononcée à l'occasion de sa retraite, après 38 ans d'engagement social.

\section{Bibliographie}

MELCHIOR, Jean-Philippe (2011). "De l'éthique professionnelle des travailleurs sociaux ", Informations sociales, Caisse nationale d'allocations familiales, p. 123-130, réf. du 2 février 2017, https:/www.cairn.info/revue-informations-sociales-2011-5-page-123.htm 